Interfaces and Free Boundaries 1, (1999) 3-16

\title{
Parallel algorithms for the solution of variational inequalities
}

\author{
JACQUES-LOUIS LIONS \\ Collège de France, 3, rue d'Ulm, 75231 Paris Cedex 05, France
}

[Received 15 May 1998]

\begin{abstract}
One of the many ways of solving free-boundary problems is, when possible, to put them (perhaps after suitable transformations) in the framework of variational or quasi-variational inequalities. It then remains to solve them numerically, a task which has been studied by Glowinski, Lions, \& Tremolieres [9] without reference to parallel algorithms.

On the other hand, systematic attempts to decompose the problems of the calculus of variations and of control theory have been made by Bensoussan, Lions, \& Temam [4], using, among other things, ideas arising from splitting methods (see Marchuk [25] and the bibliography therein).

We propose here a general method for obtaining, in infinitely many ways, stable parallel algorithms for the solution of variational inequalities of evolution. This method was introduced in [12] for equations of evolution. We show here how it can be adapted to variational inequalities (what is needed from [12] is recalled here).
\end{abstract}

\section{Introduction}

We want to introduce parallel algorithms for the analysis and control of free-surface problems. We consider here some particular free-surface problems which can be expressed in the framework of variational inequalities. The formulation of variational inequalities will be (briefly) recalled in Section 2 below. For the time being, to fix ideas, let us recall merely that the classical Stefan free-surface problem can be formulated in the framework of variational inequalities. There is a huge literature devoted to parallel algorithms. Many of them are based on domain-decomposition methods, see P. L. Lions [24] Glowinski, Periaux, Shi, Widlund [10] and the bibliography therein. We have introduced in [12], and we have begun to develop in [13]-[16], a very general formulation of decomposition methods. This method is introduced and used here for the 'parallel solution' of variational inequalities. The general (abstract) decomposition is presented in Section 3, some examples being given in Section 4. Parallel algorithms are then given in Section 5. Section 6 presents further remarks and problems. We do not study here the stabilization and control of variational inequalities using the method of decomposition of Sections 3 and 5 .

The numerical solution of variational inequalities has been studied by Glowinski, Lions, \& Tremolieres [9], but without decomposition and parallelism.

Systematic methods of decomposition of problems of the calculus of variations and of control have been introduced by Bensoussan, Lions, \& Temam [4], based on the decomposition of operators related to splitting methods (see the bibliography therein) and on the splitting of constraints, such as introduced by Lions \& Temam [23]. All these methods could be combined with those introduced here, but the benefits would be unclear. We note also that the penalty arguments used here could be replaced by Lagrange-multiplier techniques. This is developed in current work by Lions \& Pironneau [20,21]. 


\section{Formulation of the variational inequalities of evolution}

Variational inequalities of evolution were introduced by Lions \& Stampacchia [22]. We give here a simple presentation of them.

We are given two real Hilbert spaces $V$ and $H$ such that

$$
V \subset H, \quad V \text { dense in } H, \quad V \rightarrow H \text { being continuous. }
$$

We identify $H$ with its dual, so that if $V^{\prime}$ denotes the dual of $V$, then

$$
V \subset H \subset V^{\prime} \text {. }
$$

We are also given a set $K \subset V$ such that

$$
K \text { is a closed convex subset of } V \text {. }
$$

We do not restrict generality (it suffices to make a translation) by assuming that

$$
0 \in K
$$

Let $f$ be given such that

$$
f \in L^{2}\left(0, T ; V^{\prime}\right) \text {. }
$$

We consider now a bilinear form

$$
\begin{aligned}
& u, \hat{u} \rightarrow a(u, \hat{u}) \text { which is continuous on } V \times V, \\
& a(u, \hat{u}) \text { is symmetric } \text { or not, } \\
& a(u, u) \geqslant \alpha\|u\|_{V}^{2}, \quad \alpha>0, \quad \forall u \in V
\end{aligned}
$$

(where we denote by $\|u\|_{X}$ the norm of $u$ in $X$ ).

We are interested in the solution of the following variational inequalities of evolution: find $u$ such that

$$
\begin{aligned}
& u \in L^{2}(0, T ; V) \cap L^{\infty}(0, T ; H), \quad u(t) \in K \text { a.e., } \\
& \left(\frac{\partial u}{\partial t}, \hat{u}-u\right)_{H}+a(u, \hat{u}-u) \geqslant(f, \hat{u}-u) \quad \forall \hat{u} \in K, \\
& u(0)=0 .
\end{aligned}
$$

REMARK 2.1 The solution has to be thought of as being a weak solution of (2.7); otherwise the condition ' $u(0)=0$ ' in (2.7) is somewhat ambiguous. This condition becomes precise if we add the condition

$$
\frac{\partial u}{\partial t} \in L^{2}\left(0, T ; V^{\prime}\right)
$$

but this condition can be too restrictive. We can introduce weak solutions in the following form.

We consider smooth functions $\hat{u}$ such that

$$
\begin{array}{ll}
\hat{u} \in L^{2}(0, T ; V), & \frac{\partial \hat{u}}{\partial t} \in L^{2}\left(0, T ; V^{\prime}\right), \\
\hat{u}(t) \in K \text { for a.e. } t, & \hat{u}(0)=0 .
\end{array}
$$


Then, if $u$ satisfies (2.7) and is supposed to be smooth enough, we have (we write $(u, \hat{u})$ instead of $\left.(u, \hat{u})_{H}\right)$

$$
\begin{aligned}
& \int_{0}^{T}\left[\left(\frac{\partial \hat{u}}{\partial t}, \hat{u}-u\right)+a(a, \hat{u}-u)-(f, \hat{u}-u)\right] \mathrm{d} t \\
= & \int_{0}^{T}\left[\left(\frac{\partial u}{\partial t}, \hat{u}-u\right)+a(u, \hat{u}-u)-(f, \hat{u}-u)\right] \mathrm{d} t \\
& +\int_{0}^{T}\left(\frac{\partial(\hat{u}-u)}{\partial t}, \hat{u}-u\right) d t .
\end{aligned}
$$

The last term equals $\frac{1}{2}\|\hat{u}(T)-u(T)\|_{H}^{2}$ (since $u(0)=0, \hat{u}(0)=0$ ), so that

$$
\int_{0}^{T}\left[\left(\frac{\partial \hat{u}}{\partial t}, \hat{u}-u\right)+a(u, \hat{u}-u)-(f, \hat{u}-u)\right] \mathrm{d} t \geqslant 0
$$

for all $\hat{u}$ satisfying (2.9).

We then define a weak solution of (2.7) as a function $u$ such that

$$
u \in L^{2}(0, T ; V), \quad u(t) \in K \text { a.e., }
$$

and which satisfies (2.10) for all $\hat{u}$ satisfying (2.9). See Lions [17] and a simple presentation in [18].

REMARK 2.2 Let us show how formally (2.7) 'follows' from (2.10). Let us take, in (2.10),

$$
\hat{u}=\theta w+(1-\theta) u, \quad 0<\theta<1,
$$

where $w$ is smooth and satisfies conditions analogous to those of (2.9).

Then, after dividing by $\theta$, we have

$$
\int_{0}^{T}\left[\left(\theta \frac{\partial w}{\partial t}+(1-\theta) \frac{\partial u}{\partial t}, w-u\right)+a(u, w-u)-(f, w-u)\right] \mathrm{d} t \geqslant 0 .
$$

Letting $\theta \rightarrow 0$, we obtain

$$
\int_{0}^{T}\left[\left(\frac{\partial u}{\partial t}, w-u\right)+a(u, w-u)-(f, w-u)\right] \mathrm{d} t \geqslant 0
$$
for all $w \in L^{2}(0, T ; V)$ such that $w(t) \in K$ a.e. .

By taking

$$
w= \begin{cases}\hat{u} & \text { in a neighbourhood } \left.\sigma \text { of } t_{o} \in\right] 0, T[ \\ u & \text { elsewhere, }\end{cases}
$$

and letting $|\sigma| \rightarrow 0$, we obtain (2.7).

REMARK 2.3 For the proofs of existence and uniqueness of the solution of (2.7) or (2.10), we refer to Lions \& Stampacchia [22], and to the books $[17,18]$. 
REMARK 2.4 The formulation of Stefan's problem in the framework of variational inequalities is due to Duvaut [5]. For simple proofs, see for instance [18] or the recent course [28]. Very many free-boundary problems in the framework of variational inequalities are introduced and studied by Duvaut \& Lions [6], Baiocchi \& Capelo [1], Kinderlehrer \& Stampacchia [11], Elliott \& Ockendon [7], Friedmann [8], Meirmanov [26], and Rodrigues [27].

REMARK 2.5 The methods which follow apply to all the variational inequalities introduced in these references - with the exception of non-local problems: see Section 6.

\section{Decomposition method}

We introduce $N$ couples of Hilbert spaces $V_{i}$ and $H_{i}$, and $N$ convex sets $K_{i}$ :

$$
\begin{gathered}
V_{i} \subset H_{i} \subset V_{i}^{\prime} \quad(i=1, \ldots, N), \\
K_{i} \subset V_{i}, K_{i} \text { closed convex subset of } V_{i}, \text { non empty. }
\end{gathered}
$$

We are given linear operators $r_{i}$ such that

$$
\begin{aligned}
& r_{i} \in \mathcal{L}\left(H ; H_{i}\right) \cap \mathcal{L}\left(V ; V_{i}\right) \quad(i=1, \ldots, N), \\
& r_{i} \text { maps } K \text { into } K_{i} .
\end{aligned}
$$

We are also given a family of Hilbert spaces $H_{i j}$ such that

$$
H_{i j}=H_{j i} \quad \forall i, j \in[1, \ldots, N],
$$

and a family of operators $r_{i j}$ such that

$$
r_{i j} \in \mathcal{L}\left(H_{j} ; H_{i j}\right)
$$

The following hypotheses are made:

$$
r_{j} r_{j i} \varphi=r_{i} r_{i j} \varphi \quad \forall \varphi \in V
$$

if $N$ elements $u_{i}$ are given such that

$$
u_{i} \in K_{i} \quad \forall i, \quad r_{i j} u_{j}=r_{j i} u_{i} \quad \forall i, j
$$

then there exists $u \in K$ such that

$$
u_{i}=r_{i} u, \quad \text { and moreover } \quad\|u\|_{V}^{2} \leqslant c\left(\sum_{i=1}^{N}\left\|u_{i}\right\|_{V_{i}}^{2}\right) \text {. }
$$

REMARK 3.1 Examples are given in Section 4.

REMARK 3.2 If $K_{i}=V_{i}$ for all $i$, we are in the situation of equations (see $[13,14]$ ).

REMARK 3.3 The hypothesis

$$
K_{i}=V_{i} \text { for a subset of }[1, \ldots, N]
$$

is perfectly acceptable! 
We now proceed with the decomposition of the problem. We introduce the following bilinear forms:

$$
\begin{aligned}
& \quad c_{i}\left(u_{i}, \hat{u}_{i}\right) \text { is continuous, symmetric, on } H_{i} \times H_{i}, \text { and it satisfies } \\
& \quad c_{i}\left(u_{i}, u_{i}\right) \geqslant \gamma_{i}\left\|u_{i}\right\|_{H_{i}}^{2}, \gamma_{i}>0, \forall u_{i} \in H_{i}, \\
& a_{i}\left(u_{i}, \hat{u}_{i}\right) \text { is continuous, symmetric } \text { or not, on } V_{i} \times V_{i} \text {, and it satisfies } \\
& a_{i}\left(u_{i}, u_{i}\right) \geqslant \alpha_{i}\left\|u_{i}\right\|_{V_{i}}^{2}, \alpha_{i}>0, \forall u_{i} \in V_{i},
\end{aligned}
$$

We assume that

$$
\begin{array}{cc}
\sum_{i=1}^{N} c_{i}\left(r_{i} u, r_{i} \hat{u}\right)=(u, \hat{u})_{H} \quad \forall u, \hat{u} \in H, \\
\sum_{i=1}^{N} a_{i}\left(r_{i} u, r_{i} \hat{u}\right)=a(u, \hat{u}) \quad \forall u, \hat{u} \in V .
\end{array}
$$

Finally, we assume that the function $f$ is also 'decomposed' as follows:

$$
\begin{aligned}
& \text { we are given functions } f_{i} \in L^{2}\left(0, T ; V_{i}^{\prime}\right) \text { such that } \\
& \sum_{i=1}^{N}\left(f_{i}, r_{i} \hat{u}\right)=(f, \hat{u}) \quad \forall \hat{u} \in V .
\end{aligned}
$$

We are now ready to introduce the decomposed approximation.

We look for functions $u_{i}(i=1, \ldots, N)$ such that

$$
\begin{aligned}
& c_{i}\left(\frac{\partial u_{i}}{\partial t}, \hat{u}_{i}-u_{i}\right)+a_{i}\left(u_{i}, \hat{u}_{i}-u_{i}\right)+\frac{1}{\varepsilon} \sum_{j}\left(r_{j i} u_{i}-r_{i j} u_{j}, r_{j i}\left(\hat{u}_{i}-u_{i}\right)\right)_{H_{i j}} \\
& \geqslant\left(f_{i}, \hat{u}_{i}-u_{i}\right) \quad \forall \hat{u}_{i} \in K_{i}, \\
& u_{i} \in L^{2}\left(0, T ; V_{i}\right), \quad u_{i}(t) \in K_{i} \text { a.e., } \quad u_{i}(0)=0 .
\end{aligned}
$$

REMARK 3.4 Each of the variational inequalities (3.14) has to be thought of in its weak formulation, as introduced in Section 2.

REMARK 3.5 In (3.14), $\varepsilon$ is positive and small. The corresponding term in (3.14) is a penalty term.

REMARK 3.6 In the examples, $\left\|r_{j i}\right\|$ is a sparse matrix. For a given $i$, the only $j$ used in (3.14) are those such that

$$
r_{j i} \neq 0
$$

(they are the 'neighbours' of $i$ ).

One can prove 
THEOREM 3.1 The set of (decomposed) variational inequalities (3.14), (3.15) admits a unique solution $u_{i}=u_{i}^{\varepsilon}(i=1, \ldots, N)$. Further, as $\varepsilon \rightarrow 0$, one has

$$
u_{i}^{\varepsilon} \rightarrow u_{i} \quad \text { in } \quad L^{2}\left(0, T ; V_{i}\right) \text { weakly }
$$

and

$$
u_{i}=r_{i} u,
$$

where $u$ is the solution of (2.7) (actually of (2.10)).

We now present a sketch of the proof.

\section{Step 1: A priori estimates}

We can assume, without loss of generality, that $0 \in K_{i}$. Therefore taking $\hat{u}_{i}=0$ in (3.14) is allowed (for a complete proof, the technical details are much more complicated. One has to work first on approximations of (3.14), by using (other) penalty arguments; see the bibliographical references). This simplifiction gives (we write $u_{i}$ instead of $u_{i}^{\varepsilon}$ for the time being)

$$
c_{i}\left(\frac{\partial u_{i}}{\partial t}, u_{i}\right)+a_{i}\left(u_{i}, u_{i}\right)+\frac{1}{\varepsilon} X_{i} \leqslant\left(f_{i}, u_{i}\right) \quad(i=1, \ldots, N),
$$

where

$$
X_{i}=\sum_{j}\left(r_{j i} u_{i}-r_{i j} u_{j}, r_{j i} u_{i}\right)_{H_{i j}}
$$

We can write

$$
X_{i}=\frac{1}{2} \sum_{j}\left\|r_{j i} u_{i}-r_{i j} u_{j}\right\|_{H_{i j}}^{2}+\frac{1}{2} \sum_{j}\left\|r_{j i} u_{i}\right\|_{H_{i j}}^{2}-\frac{1}{2} \sum_{j}\left\|r_{i j} u_{i}\right\|_{H_{i j}}^{2} .
$$

But one verifies easily that

$$
\sum_{i} X_{i}=\frac{1}{2} \sum_{i, j}\left\|r_{j i} u_{i}-r_{i j} u_{j}\right\|_{H_{i j}}^{2} .
$$

Therefore by integration in $t$, in the interval $(0, t)$, of (3.18), and by summing in $i$, using (3.21), we obtain

$$
\begin{aligned}
& \frac{1}{2} \sum_{i} c_{i}\left(u_{i}(t)\right)+\sum \int_{0}^{t} a_{i}\left(u_{i}(s)\right) \mathrm{d} s+ \\
+ & \frac{1}{2 \varepsilon} \sum_{i, j} \int_{0}^{t}\left\|r_{j i} u_{i}(s)-r_{i j} u_{j}(s)\right\|_{H_{i j}}^{2} \mathrm{~d} s \leqslant \sum_{i} \int_{0}^{t}\left(f_{i}, u_{i}\right) \mathrm{d} s .
\end{aligned}
$$

\section{Step 2}

It follows easily from (3.22), (3.9), and (3.10) that, as $\varepsilon \rightarrow 0$ (and we now use the notation $u_{i}^{\varepsilon}$ ),

$u_{i}^{\varepsilon}$ remains in a bounded set of $L^{2}\left(0, T ; V_{i}\right) \cap L^{\infty}\left(0, T ; H_{i}\right)$,

$$
u_{i}^{\varepsilon}(t) \in K_{i}
$$




$$
\frac{1}{\sqrt{\varepsilon}}\left(r_{j i} u_{i}^{\varepsilon}-r_{i j} u_{j}^{\varepsilon}\right) \text { remains in a bounded set of } L^{2}\left(0, T ; H_{i j}\right) .
$$

Therefore we can extract a subsequence, still denoted by $u_{i}^{\varepsilon}$, such that

$$
\begin{aligned}
& u_{i}^{\varepsilon} \rightarrow u_{i} \quad \text { in } L^{2}\left(0, T ; V_{i}\right) \text { weakly, } \\
& u_{i}(t) \in K_{i},
\end{aligned}
$$

and, by virtue of (3.24), we have

$$
r_{j i} u_{i}=r_{i j} u_{j} \quad \forall i, j .
$$

Notice that we have not used the fact that $u_{i}^{\varepsilon}$ remains in a bounded set of $L^{\infty}\left(0, T ; H_{i}\right)$.

It follows from (3.25), (3.26), and the hypothesis (3.7) that

$$
u_{i}=r_{i} u_{*}, \quad u_{*}(t) \in K \text { a.e., } \quad u_{*} \in L^{2}(0, T ; V) .
$$

It remains to show that $u_{*}=u$, the solution of (2.7) or (2.10).

\section{Step 3}

We use the weak formulation recalled in Section 2. To avoid slight technical difficulties, we further weaken (2.10), by writing it (in a perfectly legitimate way!)

$$
\begin{aligned}
& \int_{0}^{T}\left[\left(\frac{\partial \hat{u}}{\partial t}, \hat{u}-u\right)+a(\hat{u}, \hat{u}-u)-(f, \hat{u}-u)\right] \mathrm{d} t \geqslant 0 \\
& \text { for all } \hat{u} \text { satisfying (2.9). }
\end{aligned}
$$

We introduce $\hat{u}_{i}$ such that

$$
\begin{aligned}
& \hat{u}_{i} \in L^{2}\left(0, T ; V_{i}\right), \quad \frac{\partial \hat{u}_{i}}{\partial t} \in L^{2}\left(0, T ; V_{i}^{\prime}\right), \\
& \hat{u}_{i}(t) \in K_{i} \text { for a.e. } t, \quad \hat{u}_{i}(0)=0,
\end{aligned}
$$

and we replace (3.24) by its (very) weak form

$$
\begin{aligned}
& \int_{0}^{T}\left[c_{i}\left(\frac{\partial \hat{u}_{i}}{\partial t}, \hat{u}_{i}-u_{i}^{\varepsilon}\right)+a_{i}\left(\hat{u}_{i}, \hat{u}_{i}-u_{i}^{\varepsilon}\right)+\frac{1}{\varepsilon} \sum_{j}\left(r_{j i} \hat{u}_{i}-r_{i j} \hat{u}_{j}, r_{j i}\left(\hat{u}_{i}-u_{i}^{\varepsilon}\right)\right)_{H_{i j}}\right] \mathrm{d} t \\
\geqslant & \int_{0}^{T}\left(f_{i}, \hat{u}_{i}-u_{i}^{\varepsilon}\right) \mathrm{d} t .
\end{aligned}
$$

Let us now assume that

$$
\begin{aligned}
& \hat{u}_{i}=r_{i} \varphi, \\
& \varphi \in L^{2}(0, T ; V), \quad \frac{\partial \varphi}{\partial t} \in L^{2}\left(0, T ; V^{\prime}\right), \quad \varphi(0)=0, \quad \varphi(t) \in K .
\end{aligned}
$$

Since $r_{j i} r_{i} \varphi=r_{i j} r_{j} \varphi$, the $\frac{1}{\varepsilon}$ terms in (3.30) drop out, so that

$$
\begin{aligned}
\int_{0}^{T}\left[c_{i}\left(\frac{\partial}{\partial t}\left(r_{i} \varphi\right), r_{i} \varphi-u_{i}^{\varepsilon}\right)\right. & \left.+a_{i}\left(r_{i} \varphi, r_{i} \varphi-u_{i}^{\varepsilon}\right)\right] \mathrm{d} t \\
& \geqslant \int_{0}^{T}\left(f_{i}, r_{i} \varphi-u_{i}^{\varepsilon}\right) \mathrm{d} t .
\end{aligned}
$$


We can pass to the limit in $\varepsilon$ in (3.32). Because of (3.27), we obtain

$$
\begin{aligned}
\int_{0}^{T}\left[c_{i}\left(\frac{\partial}{\partial t}\left(r_{i} \varphi\right), r_{i} \varphi-r_{i} u_{*}\right)\right. & \left.+a_{i}\left(r_{i} \varphi, r_{i} \varphi-r_{i} u_{*}\right)\right] \mathrm{d} t \\
& \geqslant \int_{0}^{T}\left(f_{i}, r_{i} \varphi-r_{i} u_{*}\right) \mathrm{d} t .
\end{aligned}
$$

Summing (3.33) in $i$ and using (3.11), (3.12), and (3.13), we obtain

$$
\int_{0}^{T}\left[\left(\frac{\partial \varphi}{\partial t}, \varphi-u_{*}\right)+a\left(\varphi, \varphi-u_{*}\right)-\left(f, \varphi-u_{*}\right)\right] \mathrm{d} t \geqslant 0
$$

so that (by uniqueness) $u_{*}=u$.

\section{Examples}

Let $\Omega$ be an open set of $\mathbb{R}^{d}(d=1,2,3$ in the applications). Let us consider, with the notation of Lions \& Magenes [19].

$$
\begin{gathered}
V=H_{0}^{1}(\Omega) \subset H=L^{2}(\Omega), \\
K=\{v \mid v \geqslant 0 \quad \text { in } \quad \Omega, v \in V\}, \\
(u, v)=\int_{\Omega} u v \mathrm{~d} x, \\
a(u, v)=\int_{\Omega} \nabla u \cdot \nabla v \mathrm{~d} x .
\end{gathered}
$$

For these choices of the data, variational inequalities (2.7) becomes, in an explicit form:

$$
\begin{aligned}
& \frac{\partial u}{\partial t}-\Delta u-f \geqslant 0, \\
& u \geqslant 0, \\
& \left(\frac{\partial u}{\partial t}-\Delta u-f\right) u=0 \quad \text { in } \Omega \times(0, T), \\
& u=0 \quad \text { on } \partial \Omega \times(0, T), \\
& \left.\quad u\right|_{t=0}=0 .
\end{aligned}
$$

This is (after a transformation of the unknown as by Duvaut [5]; see [18] or [28]) the Stefan problem (with one phase). The free surface (resp. free region, resp. mushy region) is defined by

$$
u=0 \quad \text { and } \quad \frac{\partial u}{\partial t}-\Delta u-f=0 \quad \text { in } \quad\{u>0\}
$$

We now decompose this problem, using the tools introduced in Section 3. We consider an overlapping covering of $\Omega$, consisting of sets $\Omega_{i}$ such that

$$
\Omega=\cup \Omega_{i},
$$


We introduce partitions of unity:

$$
\begin{aligned}
& \rho_{i} \text { are defined in } \Omega_{i}, \\
& \text { (and can be extended by } 0 \text { outside } \Omega_{i} \text { ), } \\
& \rho_{i} \geqslant 0 \quad \text { in } \quad \Omega_{i}, \\
& \sum_{i=1}^{N} \rho_{i}=1 \quad \text { in } \quad \Omega,
\end{aligned}
$$

$$
\sigma_{i} \text { has the same properties as } \rho_{i} \text {. }
$$

REMARK 4.1 One can have $\sigma_{i}=\rho_{i}$ or not. The hypothesis $\sigma_{i}=\rho_{i}$ is needed for precise error estimates, as will be shown elsewhere.

We now introduce

$$
\begin{gathered}
H_{i}=\left\{u_{i} \mid \int_{\Omega_{i}} \rho_{i} u_{i}^{2} \mathrm{~d} x<\infty\right\}, \\
V_{i}=\left\{\left.u_{i}\left|\int_{\Omega_{i}} \sigma_{i}\right| \nabla u_{i}\right|^{2} \mathrm{~d} x<\infty, u_{i}=0 \quad \text { on } \quad \partial \Omega_{i} \cap \partial \Omega\right\} .
\end{gathered}
$$

REMARK 4.2 If $\bar{\Omega}_{i} \subset \Omega$, there are no boundary conditions in (4.13). If $\partial \Omega_{i} \cap \partial \Omega \neq \emptyset$, the condition $u_{i}=0$ on $\partial \Omega_{i} \cap \partial \Omega$ does make sense.

We now define

$$
\begin{gathered}
c_{i}\left(u_{i}, \hat{u}_{i}\right)=\int_{\Omega_{i}} \rho_{i} u_{i} \hat{u}_{i} \mathrm{~d} x, \\
a_{i}\left(u_{i}, \hat{u}_{i}\right)=\int_{\Omega_{i}} \sigma_{i} \nabla u_{i} \cdot \nabla \hat{u}_{i} \mathrm{~d} x .
\end{gathered}
$$

We introduce next

$$
\begin{gathered}
H_{i j}=L^{2}\left(\Omega_{i} \cap \Omega_{j}\right), \\
r_{i} u=\text { restriction of } u \in H\left(\text { resp. V) to } \Omega_{i},\right. \\
r_{j i} u_{i}=\text { restriction of } u_{i} \in L^{2}\left(\Omega_{i}\right) \text { to } \Omega_{j} .
\end{gathered}
$$

One defines

$$
K_{i}=\left\{u_{i} \mid u_{i} \in V_{i}, u_{i} \geqslant 0 \quad \text { in } \quad \Omega_{i}\right\}
$$

Let us assume that

$$
f \in L^{2}(\Omega \times(0, T))
$$

and let $\tau_{i}$ be still another decomposition of unity. If we set

$$
f_{i}=\tau_{i} f
$$


then all the hypotheses of Section 3 are satisfied, and one can use the decomposition (3.14).

The set of inequalities (3.14) can be made explicit. Let us define

$$
P_{i}\left\{u_{1}, \ldots, u_{N}\right\}=\rho_{i} \frac{\partial u_{i}}{\partial t}-\sum_{k} \frac{\partial}{\partial x_{k}}\left(\sigma_{i} \frac{\partial u_{i}}{\partial x_{k}}\right)+\frac{1}{\varepsilon}\left(r_{j i} u_{i}-r_{i j} u_{j}\right) .
$$

Then

$$
\begin{aligned}
& P_{i}\left\{u_{1}, \ldots, u_{N}\right\}-f_{i} \geqslant 0, \\
& u_{i} \geqslant 0, \\
& \left(P_{i}\left\{u_{1}, \ldots, u_{N}\right\}-f_{i}\right) u_{i}=0 \quad \text { in } \quad \Omega_{i} \times(0, T)
\end{aligned}
$$

with

$$
u_{i}=0 \quad \text { on } \quad \partial \Omega_{i} \cap \partial \Omega
$$

and no other boundary conditions if $\sigma_{i}$ is (suitably) zero on $\partial \Omega_{i} \backslash\left(\partial \Omega_{i} \cap \partial \Omega\right)$. (Otherwise there are some Neumann boundary conditions, since the test functions do not satisfy boundary conditions outside $\partial \Omega_{i} \cap \partial \Omega$ ).

REMARK 4.3 For a given $i$, only those $j$ such that

$$
\Omega_{j} \cap \Omega_{i} \neq \emptyset
$$

appear in (4.21).

REMARK 4.4 The decomposition can be achieved in infinitely many ways.

REMARK 4.5 Several interfaces (actually $N$ ) appear. We do not know if this fact can be used to define a kind of mushy region.

REMARK 4.6 It is clear that the method presented for this particular example completely general, as far as the convex set $K$ is defined by local constraints. See Section 6 below.

REMARK 4.7 All what has been said can be adapted to non-overlapping coverings (see [13]).

We now introduce a parallel algorithm based on the decomposition method introduced in Section 3.

\section{Parallel algorithm}

We introduce the time step $\Delta t$ and a semi-discretization. We denote by $u_{i}^{n}$ (what we hope is) an approximation of $u_{i}(n \Delta t)$.

We then define $u_{i}^{n}$ by

$$
\begin{aligned}
& c_{i}\left(\frac{u_{i}^{n}-u_{i}^{n-1}}{\Delta t}, \hat{u}_{i}-u_{i}^{n}\right)+a_{i}\left(u_{i}^{n}, \hat{u}_{i}-u_{i}^{n}\right) \\
+ & \frac{1}{\varepsilon} \sum_{j}\left(r_{j i} u_{i}^{n}-r_{i j} u_{j}^{n-1}, r_{j i}\left(\hat{u}_{i}-u_{i}^{n}\right)\right)_{H_{i j}} \geqslant\left(f_{i}^{n}, \hat{u}_{i}-u_{i}^{n}\right) \quad \forall \hat{u}_{i} \in K_{i}, \\
& u_{i}^{n} \in K_{i} \quad(n=1,2, \ldots),
\end{aligned}
$$


where

$$
f_{i}^{n}=\frac{1}{\Delta t} \int_{(n-1) \Delta t}^{n \Delta t} f_{i}(t) \mathrm{d} t, \quad u_{i}^{o}=0 .
$$

REMARK 5.1 The algorithm (5.1) is parallel. Each $u_{i}^{n}$ is computed through the solution of a stationary variational inequalities. Once the $u_{j}^{n-1}$ are computed, in the computation of $u_{i}^{n}$, only those $j$ such that $r_{i j} \neq 0$ are used.

REMARK 5.2 Methods other than the penalty method could be used as well-such as Lagrangemultiplier methods. This will be reported elsewhere (see Lions \& Pironneau [21]).

Let us show now the stability of the algorithm. Replacing $\hat{u}_{i}$ by 0 in (5.1), we obtain

$$
c_{i}\left(u_{i}^{n}-u_{i}^{n-1}, u_{i}^{n}\right)+a_{i}\left(u_{i}^{n}\right)+\frac{1}{\varepsilon} X_{i}^{n} \leqslant\left(f_{i}^{n}, u_{i}^{n}\right),
$$

where

$$
X_{i}^{n}=\sum_{j}\left(r_{j i} u_{i}^{n}-r_{i j} u_{j}^{n-1}, r_{j i} u_{i}^{n}\right)_{H_{i j}}
$$

We observe that

$$
c_{i}\left(\frac{u_{i}^{n}-u_{i}^{n-1}}{\Delta t}, u_{i}^{n}\right)=\frac{1}{2 \Delta t} c_{i}\left(u_{i}^{n}-u_{i}^{n-1}\right)+\frac{1}{2 \Delta t}\left(c_{i}\left(u_{i}^{n}\right)-c_{i}\left(u_{i}^{n-1}\right)\right),
$$

so that

$$
\sum_{n=1}^{m} c_{i}\left(\frac{u_{i}^{n}-u_{i}^{n-1}}{\Delta t}, u_{i}^{n}\right)=\frac{1}{2 \Delta t} c_{i}\left(u_{i}^{m}\right)+\frac{1}{2 \Delta t} \xi_{i m}
$$

where

$$
\xi_{\text {im }}=\sum_{n=1}^{m} c_{i}\left(u_{i}^{n}-u_{i}^{n-1}\right) .
$$

We observe next that

$$
\begin{aligned}
X_{i}^{n} & =\frac{1}{2} \sum\left\|r_{j i} u_{i}^{n}-r_{i j} u_{j}^{n-1}\right\|_{H_{i j}}^{2}+\frac{1}{2} \sum_{j}\left\|r_{j i} u_{i}^{n}\right\|_{H_{i j}}^{2} \\
& -\frac{1}{2} \sum_{j}\left\|r_{i j} u_{j}^{n-1}\right\|_{H_{i j}}^{2} .
\end{aligned}
$$

If we define

$$
Y^{n}=\frac{1}{2} \sum_{i, j}\left\|r_{j i} u_{i}^{n}\right\|_{H_{i j}},
$$

then

$$
\sum_{i} X_{i}^{n}=Z^{n}+Y^{n}-Y^{n-1},
$$

where

$$
Z^{n}=\frac{1}{2} \sum_{i, j}\left\|r_{j i} u_{i}^{n}-r_{i j} u_{j}^{n-1}\right\|_{H_{i j}}^{2} .
$$


Consequently, by summing (5.3) in $i$ and in $n$, we obtain

$$
\begin{aligned}
\frac{1}{2 \Delta t} \sum_{i} c_{i}\left(u_{i}^{m}\right) & +\frac{1}{2 \Delta t} \sum_{i} \xi_{i m}+\sum_{n=1}^{m} \sum_{i} a_{i}\left(u_{i}^{n}\right) \\
& +\frac{1}{\varepsilon} Y^{m}+\frac{1}{\varepsilon} \sum_{n=1}^{m} Z^{n} \leqslant \sum_{n=1}^{m} \sum_{i}\left(f_{i}^{n}, u_{i}^{n}\right) .
\end{aligned}
$$

But

$$
\left(f_{i}^{n}, u_{i}^{n}\right) \leqslant \frac{1}{2} a_{i}\left(u_{i}^{n}\right)+\frac{c}{2}\left\|f_{i}^{n}\right\|_{V_{i}^{\prime}}^{2},
$$

so that (5.11), after multiplying by $2 \Delta t$, gives

$$
\begin{aligned}
\sum_{i} c_{i}\left(u_{i}^{m}\right) & +\sum_{i} \xi_{i m}+\Delta t \sum_{n=1}^{m} \sum_{i} a_{i}\left(u_{i}^{n}\right) \\
& +\frac{2 \Delta t}{\varepsilon} Y^{m}+\frac{2 \Delta t}{\varepsilon} \sum_{n=1}^{m} Z^{n} \leqslant c \Delta t \sum_{n=1}^{m}\left\|f_{i}^{n}\right\|_{V_{i}^{\prime}}^{2} \leqslant c_{4}
\end{aligned}
$$

hence stability follows.

REMARK 5.3 Of course other time-discretization schemes could be used in (5.1).

\section{Remarks and problems}

REMARK 6.1 An open problem. The previous methods do not apply (at least without new ideas) for nonlocal constraints - for example, for variational inequalities of the type

$$
\begin{aligned}
& \left(\frac{\partial u}{\partial t}, \hat{u}-u\right)_{H}+a(u, \hat{u}-u)+j(\hat{u})-j(u) \geqslant(f, \hat{u}-u) \quad \forall \hat{u} \in V, \\
& u(t) \in V, \quad u(0)=0,
\end{aligned}
$$

where $V=H_{0}^{1}(\Omega), H=L^{2}(\Omega)$, and where for instance

$$
j(\hat{u})=\left(\int_{\Omega}|\nabla \hat{u}|^{2} \mathrm{~d} x\right)^{\frac{1}{2}} .
$$

REMARK 6.2 Bingham's flow (see Lions \& Duvaut [6]) is an example of physical interest of variational inequalities with nonlocal constraints, similar to (but more complicated than) the previous example of Remark 6.1.

REMARK 6.3 One can extend the methods of the present paper to some quasi-variational inequalities (see [2] or [1]).

REMARK 6.4 The examples of decomposition given here (Section 4) correspond to decomposition of domains. Other possibilities can be envisioned, such as multi-Galerkin methods, using replica equations (in our case, replica variational inequalities). We shall investigate this topic in future research. Replica equations have been introduced in the paper [16], dedicated to the memory of G. Stampacchia. 


\section{REFERENCES}

1. Baiocchi, C. \& CAPElo, A. Disequazoni variazionali e quasivariazionali. Applicazioni a problemi di frontera libera, 2 volumes. Pitagora, Bologna (1978).

2. Bensoussan, A. \& Lions, J.L. Application des inéquations variationnelles en contrôle stochastique. Dunod, Paris (1978).

3. Bensoussan, A. \& Lions, J.L. Contrôle impulsionnel et inéquations quasi-variationnelles. Dunod, Paris (1982).

4. Bensoussan, A., Lions, J. L. \& Temam, R. Sur les méthodes de décomposition, de décentralisation et de coordination et applications. In: LiOns, J. L. \& MARCHUK, G. I. (eds), Méthodes mathématiques de l'informatique, pp. 133-257. Dunod, Paris (1974).

5. Duvaut, G. Résolution d'un problème de Stefan. C. R. Acad. Sci. Paris 276, (1973) 1461-1463.

6. Duvaut, G. \& Lions, J.L. Les inéquations en mécanique et en physique. Dunod, Paris (1972).

7. Elliott, C.M. \& OCKendon, J.R. Weak and variational methods for moving boundary problems Research Notes in Mathematics 59. Pitman, Boston, London (1982).

8. Friedman, A. Variational principles and free-boundary problems, Wiley, New York (1982).

9. Glowinski, R., Lions, J. L., \& Tremolieres, R. Analyse numérique des inéquations variationnelles (2 volumes). Méthodes Math. de l'Informatique. Dunod (1976).

10. Glowinski, R., Periaux, J., Shi, Z.C., \& Widlund, O. Decomposition methods in science and engineering. Wiley (to appear).

11. Kinderlehrer, D. \& Stampacchia, G. An introduction to variational inequalities and their applications. Academic Press, New York (1980).

12. Lions, J. L. Stabilization and control by parallel algorithms: An introduction. Sino-French Institute of Applied Mathematics, Fudan University, February 1998.

13. Lions, J. L. Parallel stabilization. Chinese Annals of Mathematics (1998).

14. Lions, J. L. Parallel stabilization of Navier-Stokes equations. 10th International Conference on Parallel Computational Fluid Dynamics, Hsinchu Taiwan, 11-14 May 1998.

15. Lions, J. L. Parallel stabilization of hyperbolic and Petrowsky systems. Fourth World Congress on Computational Mechanics, Buenos Aires, 29 June to 2 July 1998.

16. LiONS, J. L. Remarks on the decomposition of Navier-Stokes equations. Dedicated to the memory of G. Stampacchia, Rome, May 1998.

17. LiOnS, J. L. Quelques méthodes de résolution des problèmes aux limites non-linéaires. Dunod, Paris (1969).

18. Lions, J. L. Sur quelques questions d'analyse, de mécanique et de contrôle optimal. Les Presses de l'Université de Montréal (1976).

19. Lions, J. L. \& Magenes, E. Problemes aux limites non homogènes et applications, vol. 1. Dunod (1968).

20. Lions, J. L. \& Pironneau, O. Algorithmes parallèles pour la solution de problèmes aux limites. C. $R$. Acad. Sci. Paris 327, Ser. I (1998) 947-952.

21. Lions, J. L. \& Pironneau, O. Sur le contrôle parallèle des systèmes distribués. C. R. Acad. Sci. Paris 327, Ser. I (1998) 993-998.

22. Lions, J. L. \& Stampacchia, G. Variational inequalities. Comm. Pure Appl. Math. 20, (1967) 493-519.

23. Lions, J. L. \& Temam, R. Une méthode d'éclatement des opérateurs et des contraintes en calcul des variations. C. R . Acad. Sci. Paris 263, (1966) 563-565.

24. Lions, P. L. On the Schwarz alternating method: I, II, III. International Symposium on Domain Decomposition Methods for Partial Differential Equations. SIAM, Philadelphia, 1988, 1989, 1990.

25. MARCHUK, G. I. Methods of numerical mathematics. Springer, New York (1982). 
26. Meirmanov, A. M. The Stefan problem. Nauka, Novosibirsk (1986). English translation, De Gruyter, Berlin (1992).

27. Rodrigues, J. F. Obstacle problems in mathematical physics. North-Holland, Amsterdam (1987).

28. Rodrigues, J. F. Variational methods in the Stefan problem. Lecture Notes in Mathematics 1584, pp. 147-212. Springer, (1994). 